CCNY-HEP 4/94

May 1994

\title{
Hard Thermal Loops in a Moving Plasma and a Magnetic Mass Term
}

\author{
V.P. NAIR \\ Physics Department \\ City College of the City University of New York \\ New York, New York 10031. \\ E-mail: vpn@ajanta.sci.ccny.cuny.edu
}

\begin{abstract}
We consider the hard thermal loops of Quantum Chromodynamics for a moving quarkgluon plasma. Generalizing from this we suggest a candidate for the magnetic mass term. This mass term may also be useful in understanding the mass gap of three-dimensional non-Abelian gauge theories.
\end{abstract}


Hard thermal loops in Quantum Chromodynamics (QCD) at high temperatures have been the subject of many recent investigations [1-6]. The generating functional $\Gamma[A]$ of the hard thermal loops is a gauge-invariant nonlocal functional of the gauge potential $A_{\mu}$ and is essential in describing Debye screening and Landau damping effects as well as in carrying out the Braaten-Pisarski reorganization of perturbation theory $[1,2,3] . \Gamma[A]$ also has an elegant mathematical description, being closely related to the eikonal for a Chern-Simons (CS) theory [2].

As mentioned above, $\Gamma[A]$ is essentially an electric mass term for gluons and properly incorporating $\Gamma[A]$ in any calculation eliminates some of the infrared singularities. There would still remain some singularities since the static magnetic interactions are not screened. It is generally believed that for QCD at high temperatures there is also a magnetic mass term which screens the static magnetic interactions (or more generally magnetic fields with spacelike momenta). One way to understand how this might happen is as follows [7]. In the standard imaginary-time formalism, the partition function of QCD can be written as a Euclidean path integral with the fields periodic in the time-interval $[0, \beta=(1 / T)], T$ being the temperature. At high temperatures and for wavelengths long compared to $\beta$, the modes with nonzero Matsubara frequencies are unimportant and the theory reduces to three-dimensional QCD with a coupling constant $\sqrt{g^{2} T}, g$ being the coupling constant of the four-dimensional theory. For QCD in three dimensions we expect a mass gap $\left(\sim g^{2} T\right)$ and this is effectively the magnetic mass of the high temperature four-dimensional QCD. While this argument is suggestive, it is by no means adequate and understanding the structure of the magnetic mass term and the calculation of its value have been difficult, although many approaches have been tried $[7,8]$.

In this paper, we propose a candidate magnetic mass term. Of course, such a mass term must be gauge-invariant and parity-even. In the rest frame of the plasma, which is usually used for temperature-dependent calculations, it will not be Lorentz-invariant. However, if we include the overall motion of the plasma, we must have Lorentz invariance 
as well. We should have Lorentz invariance in this qualified sense; a mass term which is Lorentz-invariant, independently of the motion of the plasma is not acceptable. The effective fields which exhibit local behaviour at long wavelengths are presumably related to the gluon fields by nonlocal transformations and so locality is not a priori necessary for the mass term. (Recall that the electric mass term $\Gamma[A]$ is anyway nonlocal.) Finally the putative mass term must be essentially three-dimensional in consistency with the dimensional reduction argument sketched above; i.e., it should give a mass only to the spatial components of $A_{\mu}$ and in a rotationally invariant way. We try to construct a magnetic mass term based on these requirements. Our strategy is to start from hard thermal loops or $\Gamma[A]$. We first generalize it to a moving plasma. It is then easy to see, from the structure of this generalized $\Gamma[A]$, that there is another gauge-invariant mass term, which we denote by $\tilde{\Gamma}[A]$, which is also Lorentz-invariant in the qualified sense described above. Evaluating $\tilde{\Gamma}[A]$ in the rest frame of the plasma, we see that it does indeed screen static magnetic interactions. $\tilde{\Gamma}[A]$ is our candidate magnetic mass term. (A mass term which is not parityconserving has been analyzed in ref. [9]; however it does not seem to be applicable to our problem.)

While $\tilde{\Gamma}[A]$ may give the right structure for the magnetic mass, we have no computation of its coefficient or the value of the magnetic mass. Nevertheless, even just understanding the structure of the magnetic mass can be useful. At the very least, $\tilde{\Gamma}[A]$ gives a mathematically precise and gauge-invariant way of introducing an infrared cutoff in calculations of processes in the quark-gluon plasma. It may also be possible, knowing $\tilde{\Gamma}[A]$, to set up a gauge-invariant self-consistent evaluation of the magnetic mass.

The generating functional for hard thermal loops can be written as

$$
\Gamma[A]=m^{2} \int d \Omega K\left[A_{+}, A_{-}\right]
$$

where $A_{+}=\frac{1}{2} A \cdot Q, A_{-}=\frac{1}{2} A \cdot Q^{\prime}, Q^{\mu}=(1, \vec{Q}), Q^{\prime \mu}=(1,-\vec{Q}) . A_{\mu}=A_{\mu}^{a}\left(-i t^{a}\right)$ where $t^{a}$ are hermitian matrices giving a basis for the fundamental representation of the Lie algebra 
of the gauge group, here taken to be $S U(N)$. Further $\vec{Q}^{2}=1$, so that $Q^{\mu}$ and $Q^{\prime \mu}$ are null vectors, i.e., $Q^{\mu} Q_{\mu}=Q^{\prime \mu} Q_{\mu}^{\prime}=0$. The $d \Omega$-integration in (1) is over the orientations of the unit vector $\vec{Q}$. In the lowest order analysis, $m^{2}=\left(N+\frac{1}{2} N_{F}\right) \frac{T^{2}}{6}$ where $N$ is the number of colors and $N_{F}$ is the number of quark-flavors. We also have

$$
K\left[A_{z}, A_{\bar{z}}\right]=-\frac{1}{\pi} \int d^{2} x^{T}\left[\int d^{2} z \operatorname{Tr}\left(A_{z} A_{\bar{z}}\right)+i \pi I\left(A_{z}\right)+i \pi I\left(A_{\bar{z}}\right)\right]
$$

where $x^{T}$ is transverse to $\vec{Q}$, i.e. $x^{T} \cdot Q=0$. Also

$$
I\left(A_{z}\right)=i \sum_{2}^{\infty} \frac{(-1)^{n}}{n} \int \frac{d^{2} z_{1}}{\pi} \cdots \frac{d^{2} z_{n}}{\pi} \frac{\operatorname{Tr}\left(A_{z}\left(x_{1}\right) \cdots A_{z}\left(x_{n}\right)\right)}{\bar{z}_{12} \cdots \bar{z}_{n-1 n} \bar{z}_{n 1}}
$$

Here $\bar{z}_{i j}=\bar{z}_{i}-\bar{z}_{j}$ and all $A$ 's in (3) have the same argument for the transverse coordinates $x^{T}$. We have written down the Euclidean version; $K\left[A_{+}, A_{-}\right]$and $I\left(A_{+}\right)$are obtained by a simple continuation. $z$ and $\bar{z}$ denote the Euclidean version of the lightcone coordinates $\left(Q^{\prime} \cdot x\right),(Q \cdot x)$ respectively; correspondingly $A_{+}$and $A_{-}$have the Euclidean versions $A_{z}, A_{\bar{z}}$. The Minkowski version is obtained by noting that $\frac{1}{\pi} \frac{1}{\bar{z}-\bar{z}^{\prime}}$ is the Green's function for $\partial_{z}$ and continues to the Green's function

$$
G(x-y)=2 i \int \frac{d^{4} k}{(2 \pi)^{4}} e^{-i k(x-y)} \frac{1}{k \cdot Q} .
$$

$I\left(A_{-}\right)$, or its Euclidean counterpart $I\left(A_{\bar{z}}\right)$, is given by (3) with $Q \leftrightarrow Q^{\prime} . I\left(A_{z}\right)$ is related to the eikonal for a CS theory. It is also possible to write $K\left[A_{+}, A_{-}\right]$in terms of the action for a Wess-Zumino-Novikov-Witten (WZNW) theory [10].

We must now generalize (1) to a moving plasma. One can derive $\Gamma[A]$ in a moving frame by recalculating it with the appropriately generalized statistical distribution functions [11]. But it is simpler to obtain it by just using Lorentz invariance. Once we make a Lorentz transformation, the time-component of $Q_{\mu}$ is no longer 1 , so we need to consider more general null vectors. A null vector can generally be written as the product of two spinors, viz. as $u^{A} e_{A \dot{A}}^{\mu} \bar{u}^{\dot{A}}$, where $e^{\mu}=\left(1, \sigma^{i}\right), \sigma^{i}$ being the Pauli matrices and $u^{A}$ is a two-spinor, with $\bar{u}^{\dot{A}}=\overline{\left(u^{A}\right)}$, the complex conjugate of $u^{A}$. The Lorentz-invariant (or 
$S L(2, \mathbf{C})$-invariant) tensors are $\epsilon^{A B}$ and $\epsilon^{\dot{A} \dot{B}}$, with, of course, no mixing of the dotted and undotted indices. These can be used to form invariant scalar products of spinors. Although not essential, this spinor notation will be quite useful in what follows.

Rather than giving a step-by-step generalization, it is simpler to write down the manifestly Lorentz-invariant version of (1) and show that it reduces to (1) in the rest frame of the plasma. Let $p^{\mu}$ be the overall velocity vector of the plasma. The appropriate generalization of (1) is given by

$$
\Gamma[A]=2 m^{2} i \int d \mu \quad \Delta(u, v) \frac{K\left(A_{u}, A_{v}\right)}{(u \cdot v)(\bar{u} \cdot \bar{v})}
$$

where we have two sets of two-component spinors $\left(u^{A}, \bar{u}^{\dot{A}}\right)$ and $\left(v^{A}, \bar{v}^{\dot{A}}\right)$ (see Eq. (9) for an explicit parametrization) and

$$
\begin{gathered}
A_{u}=\frac{1}{2} u^{A}(A \cdot e)_{A \dot{A}} \bar{u}^{\dot{A}}, \quad A_{v}=\frac{1}{2} v^{A}(A \cdot e)_{A \dot{A}} \bar{v}^{\dot{A}} \\
d \mu=\frac{u \cdot d u \quad \bar{u} \cdot d \bar{u} \quad v \cdot d v \bar{v} \cdot d \bar{v}}{(u \cdot v)^{2}(\bar{u} \cdot \bar{v})^{2}} \\
\Delta(u, v)=(u \cdot v)(\bar{u} \cdot \bar{v}) \delta(v(p \cdot e) \bar{u}) \delta(u(p \cdot e) \bar{v})
\end{gathered}
$$

Here $u \cdot d u=u^{1} d u^{2}-u^{2} d u^{1}$, etc. The measure $d \mu$ and the generalized $\delta$-function $\Delta(u, v)$ are invariant under complex rescalings of $u, v$, i.e., under $u \rightarrow \lambda_{1} u, v \rightarrow \lambda_{2} v$. The space of spinors is thus two copies of $\mathbf{C} P^{1}=S^{2}$ with an identification between the two enforced by the $\delta$-functions. This identification depends on $p^{\mu}$ and hence is frame-dependent. Expression (5) is manifestly Lorentz-invariant if one transforms $p^{\mu}$ as well.

We now evaluate (5) in the rest frame of the plasma. Introduce the parametrization

$$
\begin{array}{ll}
u=\rho\left(\begin{array}{c}
1 \\
z
\end{array}\right) & \bar{u}=\bar{\rho}\left(\begin{array}{c}
1 \\
\bar{z}
\end{array}\right) \\
v=\sigma\left(\begin{array}{c}
\bar{w} \\
-1
\end{array}\right) & \bar{v}=\bar{\sigma}\left(\begin{array}{c}
w \\
-1
\end{array}\right)
\end{array}
$$

We then have

$$
d \mu=\frac{d z d \bar{z} d w d \bar{w}}{(1+z \bar{w})^{2}(1+\bar{z} w)^{2}}
$$


With $p^{\mu}=(1,0,0,0)$, the $\Delta$-function becomes $\Delta(u, v)=(1+\bar{z} w)(1+z \bar{w}) \delta(w-z) \delta(\bar{w}-\bar{z})$. Further $A_{u}=\frac{1}{2}(A \cdot Q) \rho \bar{\rho}(1+z \bar{z}), \quad A_{v}=\frac{1}{2}\left(A \cdot Q^{\prime}\right) \sigma \bar{\sigma}(1+w \bar{w})$ where

$$
Q^{\mu}=\left(1, \frac{z+\bar{z}}{(1+z \bar{z})}, \frac{i(z-\bar{z})}{(1+z \bar{z})}, \frac{1-z \bar{z}}{(1+z \bar{z})}\right) \equiv(1, \vec{Q}(z))
$$

and $Q^{\prime \mu}=(1,-\vec{Q}(w))$. The standard parametrization of $\vec{Q}$ in terms of the direction cosines is obtained if we write $z=e^{-i \varphi} \tan (\theta / 2) . \rho, \sigma$-dependence cancels out since $K$ is homogeneous of degree two in these variables. Integrating out the $\delta$-functions, we see that (5) indeed becomes (1) in the rest frame. One can in a straightforward manner evaluate the $n$-point functions in an arbitrary frame; the expressions, even for the two-point function, are too long to be displayed here.

We now consider alternatives to (5). The possible changes are in the $\delta$-functions or in the combinations $A_{u}, A_{v}$. For the argument of the $\delta$-functions, we can use $v(p \cdot e) \bar{u}$ as we have done or $v(p \cdot e) \bar{v}$ (and their conjugates). The second choice implies the vanishing of $v^{A}$ in the rest frame and is not acceptable. The only modification is thus in the combinations for the gauge potential. We can have (6) or

$$
\tilde{A}_{u}=\frac{1}{2} u^{A}(A \cdot e)_{A \dot{A}} \bar{v}^{\dot{A}}, \quad \tilde{A}_{v}=\frac{1}{2} v^{A}(A \cdot e)_{A \dot{A}} \bar{u}^{\dot{A}}
$$

We thus consider the term

$$
\tilde{\Gamma}[A]=\left(-2 M^{2} i\right) \int d \mu \quad \Delta(u, v) \frac{K\left[\tilde{A}_{u}, \tilde{A}_{v}\right]}{(u \cdot v)(\bar{u} \cdot \bar{v})}
$$

In the rest frame of the plasma this simplifies to

$$
\tilde{\Gamma}[A]=-M^{2} \int d \Omega K\left[A_{n}, A_{\bar{n}}\right]
$$

where $A_{n}=\frac{1}{2} A_{i} n_{i}, A_{\bar{n}}=\frac{1}{2} A_{i} \bar{n}_{i}$ and $n_{i}=(-\cos \theta \cos \varphi-i \sin \varphi,-\cos \theta \sin \varphi+i \cos \varphi, \sin \theta)$, using $z=e^{-i \varphi} \tan (\theta / 2)$. Notice that $n_{i}$ is a complex three-dimensional null vector. $\tilde{\Gamma}[A]$ involves, in the rest frame, only the spatial components of $A_{\mu}$ as expected for a magnetic 
mass term. $I\left(A_{n}\right), I\left(A_{\bar{n}}\right)$ are defined using $n \cdot x$ and $\bar{n} \cdot x$ in place of $z, \bar{z}$ in (3). Thus $K$ in (14) is $K\left[\frac{1}{2} A \cdot Q, \frac{1}{2} A \cdot Q^{\prime}\right]$ of $(2,3)$ with $Q^{\mu} \rightarrow\left(0, n_{i}\right), Q^{\prime \mu} \rightarrow\left(0, \bar{n}_{i}\right)$.

Consider the simplification of the quadratic term in (14). Using $\int d \Omega n_{i} \bar{n}_{j}=\frac{8 \pi}{3} \delta_{i j}$ and

$$
\int d \Omega \frac{k \cdot \bar{n}}{k \cdot n} n_{i} n_{j}=\frac{8 \pi}{3}\left[\frac{k_{i} k_{j}}{\vec{k}^{2}}-\frac{1}{2}\left(\delta_{i j}-\frac{k_{i} k_{j}}{\vec{k}^{2}}\right)\right]
$$

we find

$$
\tilde{\Gamma}[A]=-\frac{M^{2}}{2} \int \frac{d^{4} k}{(2 \pi)^{4}} A_{i}^{a}(-k)\left(\delta_{i j}-\frac{k_{i} k_{j}}{\vec{k}^{2}}\right) A_{j}^{a}(k)+\mathcal{O}\left(A^{3}\right)
$$

Thus $\tilde{\Gamma}[A]$ does give screening of transverse magnetic interactions, with a screening mass $M$.

The higher order terms in $\tilde{\Gamma}[A]$ can also be evaluated in a straightforward fashion, noting that the basic change is replacing $Q^{\mu}$ by $\left(0, n_{i}\right)$ and $Q^{\prime \mu}$ by $\left(0, \bar{n}_{i}\right)$. ( Expressions in $n_{i}, \bar{n}_{i}$ which arise from the projectively invariant spinorial expressions lead to rotationally invariant results. As a word of caution, an arbitrary expression involving the $n_{i}, \bar{n}_{i}$ cannot be written in the spinor language without explicitly breaking rotational symmetry. Such a complication, however, does not arise for $\tilde{\Gamma}[A]$.) Notice also that $\tilde{\Gamma}[A]$ is explicitly real, eventhough $A_{n}, A_{\bar{n}}$ are complex even in Minkowski space. In writing out $\tilde{\Gamma}[A]$, we need the inverses of $n \cdot \partial$ and $\bar{n} \cdot \partial$. Essentially only the spatial coordinates are involved and the boundary condition is the vanishing of the Green's function at infinity. The derivatives $n \cdot \partial, \bar{n} \cdot \partial$ behave like $\partial_{z}, \partial_{\bar{z}}$ with Green's functions which are the analogues of $\left(\bar{z}-\bar{z}^{\prime}\right)^{-1},\left(z-z^{\prime}\right)^{-1}$. In particular, we do not need an $i \epsilon$-prescription to define the inverses.

The various terms in $\tilde{\Gamma}[A]$ are clearly nonlocal. Just as in the case of hard thermal loops [3,4], it is possible to introduce auxiliary field variables and write the equations of motion in a local way. The equations of motion can be written as

$$
\begin{aligned}
\left(D_{\mu} F^{\mu \nu}\right)^{a}-J^{\nu a} & =0 \\
\partial_{+} \mathcal{A}_{-}-\partial_{-} A_{+}+\left[A_{+}, \mathcal{A}_{-}\right] & =0
\end{aligned}
$$




$$
\begin{aligned}
J^{\nu a} & =\frac{M^{2}}{2 \pi} \int d \Omega \operatorname{Tr}\left\{\left(-i t^{a}\right)\left[\left(\mathcal{A}_{-}-A_{-}\right) Q^{\nu}+\left(\mathcal{A}_{+}-A_{+}\right) Q^{\prime \nu}\right]\right\} \\
& =\frac{M^{2}}{2 \pi} \int d \Omega \operatorname{Tr}\left\{\left(-i t^{a}\right)\left[H^{-1} D_{-} H Q^{\nu}-D_{+} H H^{-1} Q^{\prime \nu}\right]\right\}
\end{aligned}
$$

where $D_{\mu}$ denotes the covariant derivative and $\mathcal{A}_{+}=H A_{+} H^{-1}-\partial_{+} H H^{-1}, \mathcal{A}_{-}=$ $H^{-1} A_{-} H+H^{-1} \partial_{-} H . H$ is the auxiliary matrix field, which is hermitian in the present case. These equations have the same form as the equations with the hard thermal loop contributions; the difference is that $Q^{\mu}, Q^{\prime \mu}$ are now given by $\left(0, n_{i}\right),\left(0, \bar{n}_{i}\right)$.

We can also write $n \cdot A=-n \cdot \partial U U^{-1}, \bar{n} \cdot A=U^{\dagger-1} \bar{n} \cdot \partial U^{\dagger}$ where $U$ is an $S L(N, \mathbf{C})$ matrix. In this parametrization, $\tilde{\Gamma}[A]$ becomes

$$
\tilde{\Gamma}[A]=M^{2} \int d \Omega d x^{0} d x^{T} S_{W Z N W}(G)
$$

where

$$
S_{W Z N W}(G)=-\frac{1}{2 \pi} \int_{\mathcal{M}^{2}} d^{2} x \operatorname{Tr}\left(\partial_{+} G G^{-1} \partial_{-} G G^{-1}\right)+\frac{1}{12 \pi} \int_{\mathcal{M}^{3}} \operatorname{Tr}\left(d G G^{-1}\right)^{3}
$$

In Eq.(19), $G$ is the hermitian matrix $U^{\dagger} U$ and the integration is over the directions transverse to $n_{i}, \bar{n}_{i}$, i.e., over $x^{0}$ and the spatial direction $x^{T}$. Notice that we can also write

$$
\begin{aligned}
\frac{1}{4} F_{i j}^{a} F_{i j}^{a} & =-\frac{3}{4 \pi} \int d \Omega \operatorname{Tr}\left(F_{n \bar{n}} F_{\bar{n} n}\right) \\
& =-\frac{3}{4 \pi} \int d \Omega \operatorname{Tr}\left[\partial_{\bar{n}}\left(\partial_{n} G G^{-1}\right) \partial_{n}\left(\partial_{\bar{n}} G G^{-1}\right)\right]
\end{aligned}
$$

This result also shows that the action for (Euclidean) three-dimensional gauge theory, with the mass term added, can be written as

$$
\begin{aligned}
\mathcal{S}^{(3)} & =\int d \Omega d x^{T} \mathcal{S}^{(2)} \\
\mathcal{S}^{(2)} & =-\frac{3}{4 \pi} \int d^{2} z \operatorname{Tr}\left(F_{n \bar{n}} F_{\bar{n} n}\right)-M^{2} S_{W Z N W}(G)
\end{aligned}
$$

$\mathcal{S}^{(2)}$ is the action for two-dimensional QCD with an extra WZNW-action; the fields do depend on all three coordinates but the transverse coordinate $x^{T}$ only plays the role of a parameter as far as $\mathcal{S}^{(2)}$ is concerned. This representation may be useful in understanding 
the mass gap in three-dimensional gauge theories. Of course, as we have mentioned before, we do not have a calculation of $M^{2}$, either for QCD at finite temperature or intrinsically for the three-dimensional gauge theory. In principle, one should not have to add such a mass term, it should emerge from a calculation of the effective action. A possible alternative, knowing the structure of this mass term, is to seek a self-consistent evaluation of $M^{2}$. In the context of the magnetic mass for the QCD plasma, such an approach has been attempted in reference [8]. With our mass term, this calculation can perhaps be refined, especially regarding questions of gauge invariance.

I thank R.Jackiw and D.Minic for useful discussions.

\section{References}

[1] R. Pisarski, Physica A 158, 246 (1989); Phys.Rev.Lett. 63, 1129 (1989); E. Braaten and R. Pisarski, Phys.Rev. D 42, 2156 (1990); Nucl.Phys. B 337, 569 (1990); ibid. B 339, 310 (1990); Phys.Rev. D 45, 1827 (1992); J. Frenkel and J.C. Taylor, Nucl.Phys. B 334, 199 (1990); J.C. Taylor and S.M.H. Wong, Nucl.Phys. B 346, 115 (1990).

[2] R. Efraty and V.P. Nair, Phys.Rev.Lett. 68, 2891 (1992); Phys.Rev. D 47, 5601 (1993).

[3] R. Jackiw and V.P. Nair, Phys.Rev. D 48, 4991 (1993)

[4] V.P. Nair, Phys.Rev. D 48, 3432 (1993); CCNY Preprint 2/94, March 1994 (to appear in Phys. Rev.D).

[5] J.P. Blaizot and E. Iancu, Phys.Rev.Lett. 70, 3376 (1993); Saclay Preprint T93/064 (to appear in Nucl.Phys. B); Saclay Preprints T94/02,03, 013 (1994).

[6] R. Jackiw, Q. Liu and C. Lucchesi, MIT preprint CTP\#2261 (1993); P.F. Kelly, Q. Liu, C. Lucchesi and C. Manuel, MIT Preprint CTP\#2292, March 1994. 
[7] A.D. Linde, Phys.Lett. B 96, 289 (1980); D. Gross, R. Pisarski and L. Yaffe, Rev.Mod.Phys. 53, 43 (1981).

[8] O.K. Kalashnikov, JETP Lett. 39, 405 (1984).

[9] S.M. Carroll, G.B. Field and R. Jackiw, Phys.Rev. D 41, 1231 (1990).

[10] S.P. Novikov, Usp.Mat.Nauk 37, 3 (1982); E. Witten, Commun.Math.Phys. 92, 455 (1984).

[11] S.R. de Groot, W.A. van Leeuwen and C.G. van Weert, Relativistic Kinetic Theory: Principles and Applications, North Holland, New York (1980). 